\title{
PROPORCIONES DE LAS PIEZAS DENTARIAS
}

\section{Del sector anterosuperior en relación a la sección divina en estudiantes de la Escuela Profesional de Odontología, UNSAAC 2014.}

Nelly Valdez Aduviri', Yahaira Paola Vargas Gonzales², Juan Carlos Romero Diaz ${ }^{3}$.

RESUMEN

El conocimiento de la sección divina se descubrió hace siglos y admiró a grandes científicos por ser una constante matemática increíblemente perfecta y repetible; sin embargo, no fue sino hasta mediados del siglo XIX que se llegó a la conclusión de que el cuerpo humano y la naturaleza pueden ser descritos por dicha constante matemática. Los profesionales del área médica y odontológica que trabajan con la función y la belleza de la cara han tratado de establecer sistemas repetibles y cuantificables que fundamenten un buen diagnóstico y un plan de tratamiento seguro. El presente estudio tuvo como objetivo: Determinar la presencia de la sección divina en las piezas dentarias del sector antero superior en los estudiantes de la Escuela Profesional de Odontología, Universidad Nacional de San Antonio Abad del Cusco 2014. Fue un estudio descriptivo, prospectivo, transversal a una muestra de 147

1 Docente Auxiliar de la Escuela Profesional de Odontología de la Universidad Nacional de San Antonio Abad del Cusco.

2 Doctora en Ciencias. Docente Auxiliar de la Escuela Profesional de Odontología de la Universidad Nacional de San Antonio Abad delCusco.E-mail:yhajaod@hotmail.com

3 Cirujano Dentista egresado de la Escuela Profesional de Odontología de la Universidad Nacional de San Antonio Abad del Cusco. 
estudiantes de la Escuela Profesional de Odontología, Resultados: La prevalencia de la sección divina en los estudiantes de la Escuela Profesional de Odontología fue $23.1 \%$ de estudiantes que si cumplieron con la sección divina, seguido de un $76,9 \%$ que no cumplieron con la sección divina, los valores cercanos de la sección divina en las comparaciones que se hizo entre las piezas 1.1/1.2, 1.2/1.3, 2.1/2.2, 2.2/2.3 fueron valores estadísticamente significativos según el rango establecido, la sección divina según la hemiarcada superior fue de un $46.26 \%$, finalmente la sección divina fue mayor en el sexo femenino en un $12.24 \%$. Conclusiones: La prevalencia de la sección divina en los estudiantes de la Escuela Profesional de Odontología fue baja y mayor en el sexo femenino.

PALABRAS CLAVES: sección divina, estética.

\section{ABSTRACT}

The knowledge of the divine section was discovered centuries ago and admired great scientists to be an incredibly perfect and repeatable mathematical constant; however, it was not until the mid-nineteenth century that concluded that the human

"Sabiendo donde está la desproporción, el desequilibrio, ese problema se puede tratar. Lo importante es que los conocimientos sobre la sección divina tienen valor para todos los profesionales, desde el Dentista general, Prostodoncista, Ortodoncista; hasta el cirujano plástico y maxilofacial."

body and nature can be described by this mathematical constant. The medical and dental professionals working area with the function and beauty of the face have tried to establish repeatable and measurable systems in support of a good diagnosis and treatment insurance plan. This study aimed to: determine the presence of the divine section in the upper anterior teeth sector students career of Dentistry, Universidad Nacional de San Antonio Abad del Cusco 2014. It was a descriptive, prospective, cross-sectional study of a sample of 147 students Dental career, Results: The prevalence of divine section students career of Dentistry was $23.1 \%$ of students met whether divine section, followed by $76.9 \%$ who did not meet the divine section, nearby values ??of divine section in the comparisons made between pieces $1.1 / 1.21 .2$ / $1.32 .1 /$ 2.22 .2 / 2.3 were statistically significant values ??according to the set range, divine section along the top hemiarcada was one $46.26 \%$, finally the divine section was greater in females in $12.24 \%$.Conclusions: The prevalence of divine section students of the Career of Dentistry was low and increased in females.

KEYWORDS: divine section, aesthetics a sección divina es la proporción simétrica Lerfecta del cuerpo humano, es una relación armoniosa utilizada desde la antigüedad. Es así, que en una dentición perfecta de dientes sanos, proporcionales y bien alineados, no solo manifiesta un excelente estado dentario, sino que indica un nivel inmejorable de salud general y de vitalidad. El aspecto estético en el sector dental antero superior depende fundamentalmente de la simetría y proporcionalidad de los dientes que lo constituyen.

En general, la estética va asociada a la proporcionalidad y la armonía, siendo considerado los rasgos faciales y dentales desproporcionados y asimétricos como antiestético; mientras que los proporcionados, son aceptables, aunque no siempre bellos. (1)

Sabiendo donde está la desproporción, el desequilibrio, ese problema se puede tratar. Lo importante es que los conocimientos sobre la sección divina tienen valor para todos los profesionales, desde el Dentista general, Prostodoncista, Ortodoncista; hasta el cirujano plástico y maxilofacial. Hoy en día, en odontología es necesario contar con una técnica científica, sencilla y práctica para evaluar la estética dental y facial, lo constituye aquel que se basa en el empleo de la sección divina. Uno de los métodos más polémicos y estudiados en la actualidad para el examen de la estética. (2)

Es por ello que la presente investigación tuvo como objetivo: Determinar las proporciones de las piezas dentarias del sector antero superior en relación a la sección divina en los estudiantes de la Escuela Profesional de Odontología, Universidad Nacional de San Antonio Abad del Cusco 2014.

\section{MATERIALES Y MÉTODOS}

Estudio descriptivo, prospectivo y transversal, cuya muestra estuvo constituida por 147 estudiantes de la Escuela Profesional de Odontología, debidamente matriculados y que deseaban participar en el estudio. Para el estudio se utilizó fotografías digitales y una ficha de recolección de datos. 


\section{RESULTADOS}

De un total de 147 estudiantes de la Escuela Profesional de Odontología, se encontró que aproximadamente solo un

Gráfico 1. Presencia de sección divina en los estudiantes de la Escuela Profesional de Odontología - UNSAAC 2014.

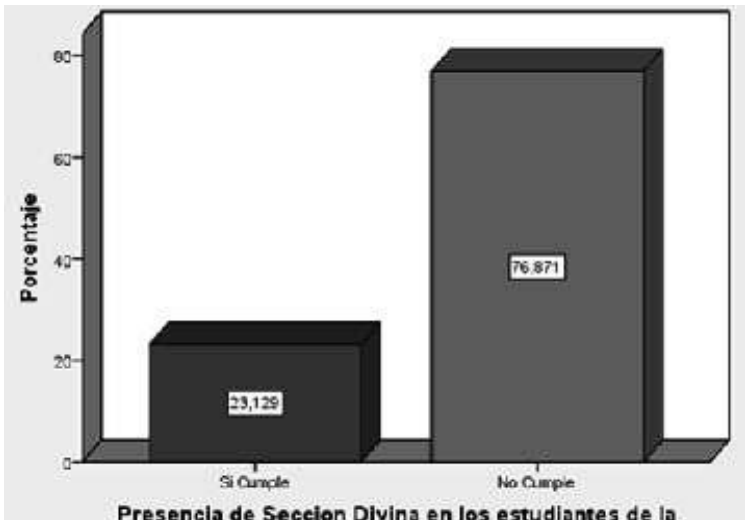

Presencia de Seccion Divina en los estudiantes de lia
Carrera Profesional de Odontologia - UNSAAC 2014

Fuente: Ficha de recolección de datos.

Gráfico 2. Presencia de la sección divina en la hemiarcada superior derecha.

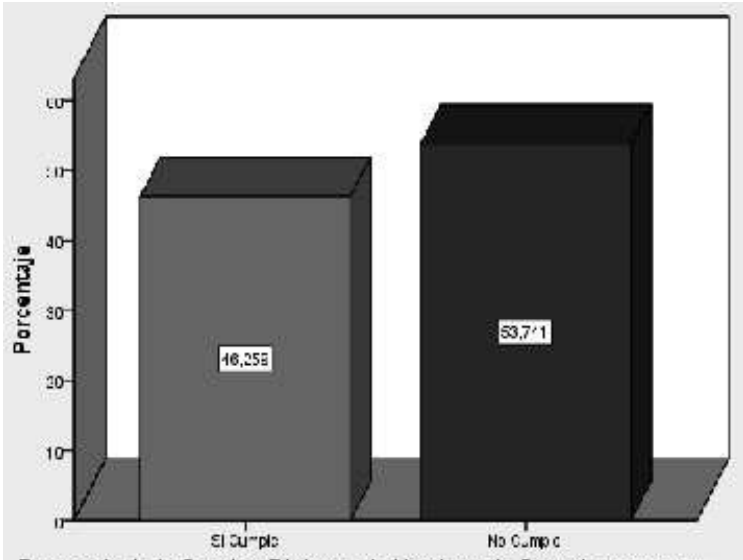

Presencla de la Secclon Divina en la Hemlarcada Superlor Darocha

Fuente: Ficha de recolección de datos.

Gráfico 3. Presencia de la sección divina en la hemiarcada superior izquierda.

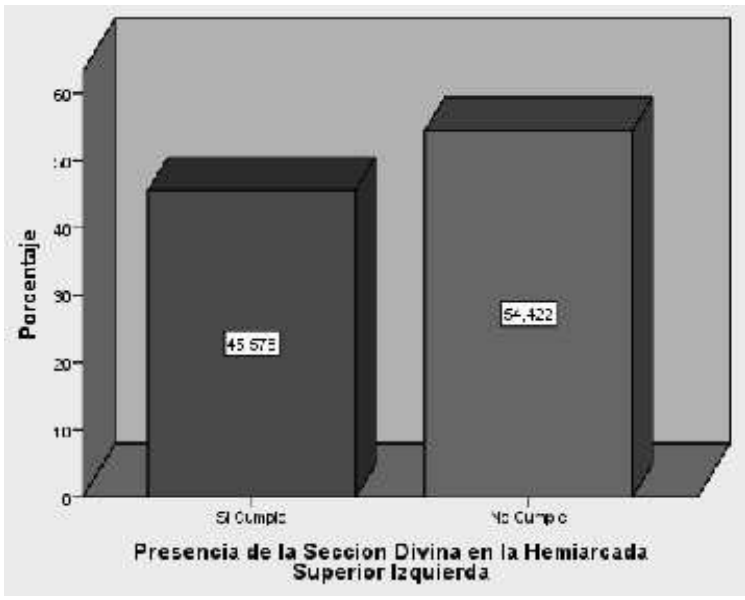

Fuente: Ficha de recolección de datos. cuarto de la población total presentaban la sección divina con un porcentaje de $23.1 \%$ equivalente a 34 estudiantes, seguido de un $76.9 \%$ que representaba 113 estudiantes que no cumplían con la sección divina.

Gráfico 4. Presencia de sección divina en los estudiantes de la Escuela Profesional de Odontología UNSAAC - 2014, según sexo.

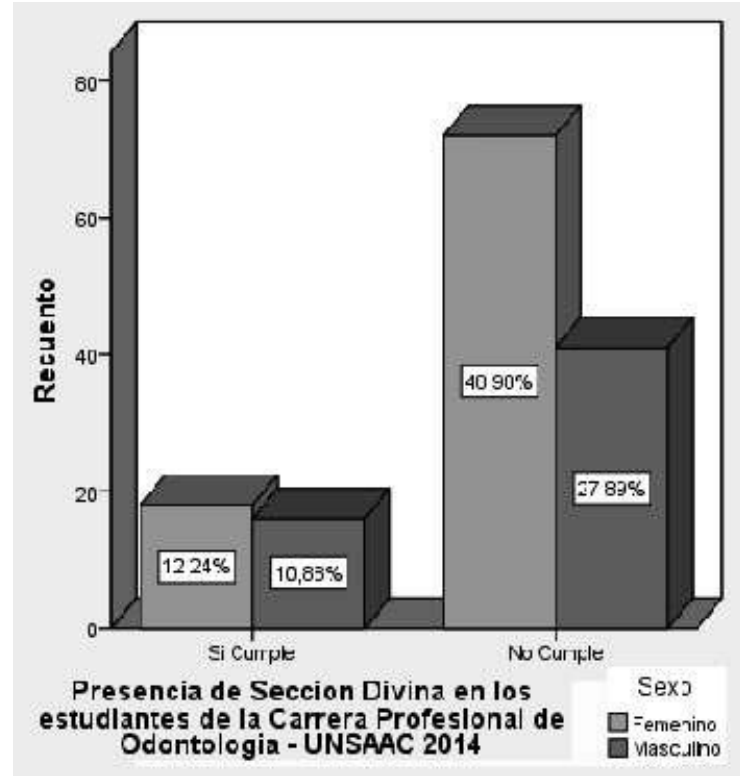

Fuente: Ficha de recolección de datos.

\section{DISCUSIÓN}

La presencia de la sección divina en las piezas dentarias del sector antero superior, en los estudiantes de la Escuela Profesional de Odontología de la Universidad Nacional de San Antonio Abad del Cusco fue del 23.1\%, quienes presentaron sus piezas dentarias antero superiores en sección divina; resultados que difieren del estudio realizado por Ribeiro I. (75.8\%), esto puede deberse a que dicho estudio se realizó en una población con características genotípicas diferentes, y que hubo mayor predominio de población masculina. También en el presente estudio se encontró que en las relaciones de proporcionalidad del ancho mesio distal de las piezas 1.1/1.2, 1.2/1.3, 2.1/2.2, 2.2/2.3 se obtuvieron valores promedios de 1.720 , $1.669,1.719,1.664$ respectivamente y que se encuentran dentro del rango de la sección divina que se estableció de $1.5-1.75$, estudio similar al realizado por Zapata $C$. en las que se encontró valores de $1.462,1.217,1.464 \mathrm{y}$ 1.220 respectivamente. 
También se hizo un análisis de la sección divina según el sexo en el cual se encontró que, del total de los estudiantes que presentan sección divina el $12,2 \%$ son del sexo femenino, y el $10.9 \%$ son del sexo masculino, comparando con el estudio realizado por Vargas $M$. en el que se encontró la sección divina en $26 \%$ en sexo femenino y un $18.8 \%$ en el sexo masculino, podemos decir que los valores obtenidos en el presente trabajo fueron diferentes, pero que el predominio de la sección divina en ambos trabaj os comparados tienen preferencia por el sexo femenino.

Según el estudio de Gallao S. podemos comentar que la presencia de la sección divina es evidente en todas las piezas dentarias del sector anterior, mencionando que la simetría de las medidas dentarias en la arcada superior fue perfecta, de lo que podemos decir que según el presente estudio también se encontró un valor significativo de presencia de proporcionalidad y simetría en las piezas dentarias del sector antero superior.

Y por último, el estudio realizado por Tito R. determinó la presencia de la sección divina en los estudiantes de la Universidad Nacional Jorge Basadre Grohmann de Tacna, en la cual se demostró que existía presencia de la sección divina, con un valor significativo; y de la cual podemos afirmar según el presente estudio que la presencia de la sección divina es evidente en los estudiantes universitarios de dicha universidad.

\section{CONCLUSIONES}

1. Las proporciones de las piezas dentarias del secto $r$ anter o superior de los estudiantes de la Escuela Profesional de Odontología, presentaron la sección divina de acuerdo al rango establecido.

2. Los valores promedios de relacionar las piezas dentarias del sector antero superior

$1.1 / 1.2, \quad 1.2 / 1.3, \quad 2.1 / 2.2 \quad \mathrm{y}$ $2.2 / 2.3$; y que dieron como resultado: 1.720 ,

1.669, 1.719

respectivamente, cumplieron con el rango establecido para la sección divina de 1.5 a 1.75 .

3. La hemiarcada derecha e izquierda del sector antero superior presentaron la sección divina.

4. La presencia de la sección divina fue mayor en el sexo femenino con respecto al sexo masculino.

\section{REFERENCIAS}

1. Moncada G., Pablo Angel A. "Parámetros para la evaluación de la estética dentaria superior", Revista dental de Chile 2008. [http://www.revistadentalde chile.cl/temas].

2. Marcuschamer Miller, A. "Proporción áurea en odontología", 1era Edición; Editorial: Trillas, 2012.

3. Parodi G., Corts J. "Actas Odontológicas", revista de La facultad de odontología de la universidad católica de Uruguay, [http// www.actasodontologicas. ucu.edu.uy/]

4. Garcia E., Momose T., Mongruel O., Gomes J. "Aplicación clínica de los parámetros estética en Odontología restauradora", [http://www.actaodonto logica.com/ediciones/2008/1/aplicacion clínica parámetros estéticos odontología restauradora.asp].

5. Ribeiro I. "Prevalencia de la proporción aurea en la dentición natural" Brasil 2003.

6. Zapata C. "Análisis de los Dientes Antero Superiores y su Relación con la Proporción Aurea en un grupo de Estudiantes de la Universidad de Talca", Universidad de Talca, Talca - Chile 2006.

7. Tito R. "Proporción aurea y parámetros faciales en estudiantes de la Universidad Nacional Jorge Basadre Grohmann, Tacna 2011", Tacna - Perú 2011.

8. Balsells E., Camps D., Ustrell J. "Evaluación de la sonrisa" Anales de Odontoestomatologia, Universidad de Barcelona, España 1996. [http://www.diposit.ub.edu]

9. Gallao S. "Análisis fotográfica de la simetría y de la proporción estética de los dientes anteriores", Brasil 
2009.

10. Vargas M. "Frecuencia de la proporciona Áurea en el grupo dentario antero superior en pacientes de 20 a

25 años de la clínica odontológica de la universidad católica de santa María" Arequipa - Perú 2009.

11. Livio, M. "La proporción áurea, la historia de Phi. El número más sorprendente del mundo"; Traductor Daniel Aldea Rossell e Irene Muzás Calpe, 1a Edición. Editorial Ariel, S.A. Pág 830. Barcelona - España 2009.

12. Paccioli L. "La divina proporción", Traducción del

Italiano de la Edición de 1509: Editorial, Losada. Pg:

55. Buenos Aires - Argentina 1946.

13. Dell'acqua A. "Estética en odontología restauradora", Editorial Médica Ripano, Pág: 32-17. Madrid - España 2006.

14. Jefferson Y. "Facial beauty - Establishing a universal Standard", Vol: 15. Disponible en [http://www.facial beauty.org/article/FacialBeauty.pdf], Estados Unidos 2004.

15. Acuña L. "Su Salud y Estética en Odontología", 